\title{
Optimality for Multi-Objective Programming Involving Arcwise Connected d-Type-I Functions*
}

\author{
Guolin Yu, Min Wang \\ Research Institute of Information and System Computation Science, The North University for Ethnics, \\ Yinchuan, China \\ E-mail: guolin_yu@126.com \\ Received April 26, 2011; revised May 27, 2011; accepted June 11, 2011
}

\begin{abstract}
This paper deals with the optimality conditions and dual theory of multi-objective programming problems involving generalized convexity. New classes of generalized type-I functions are introduced for arcwise connected functions, and examples are given to show the existence of these functions. By utilizing the new concepts, several sufficient optimality conditions and Mond-Weir type duality results are proposed for non-differentiable multi-objective programming problem.
\end{abstract}

Keywords: Multi-Objective Programming, Pareto Efficient Solution, Arcwise Connected d-Type-I Functions, Optimality Conditions, Duality

\section{Introduction}

Investigation on sufficiency and duality has been one of the most attraction topics in the theory of multi-objective problems. It is well known that the concept of convexity and its various generalizations play an important role in deriving sufficient optimality conditions and duality results for multi-objective programming problems. The concept of type-I functions was first introduced by Hanson and Mond [1] as a generalization of convexity. With and without differentiability, the type-I functions were extended to several classes of generalized type-I functions by many researchers, and sufficient optimality criteria and duality results are established for multi-objective programming (vector optimization) problems involving these functions (see [1-12]). Another meaningful generalization of convex functions is the introduction of arcwise connected functions, which was given by Avriel and Zang [13]. Singh [14] and Mukherjee and Yadav [15] discussed some properties of arcwise connected sets and functions. Bhatia and Mehara [16] investigated some properties of arcwise connected functions in terms of their directional derivatives and established optimality conditions for scalar-valued nonlinear programming prblems involving these functions. Mehar and Bhatia [17]

\footnotetext{
*This research is supported by Zizhu Science Foundation of Beifang University of Nationalities; Natural Science Foundation for the Youth (No. 10901004).
}

and Davar and Mehra [18] studied optimality conditions and duality results for minmax problems and fractional programming problems involving arcwise connected and generalized arcwise connected functions, respectively.

In this paper, we first introduce new classes of generalized convex type-I functions by relaxing definitions of arcwise connected function and type-I function. We present some sufficient optimality conditions and dual theorems for non-differential multi-objective programming problem under various generalized convex type-I functions assumptions. This paper is divided into four sections. Section 2 recalls some definitions and related results which will be used in later sections, and introduces new classes generalized convex type-I functions. In Section 3 and Section 4, the sufficient optimality conditions and Mond-Weir type duality results are established for non-differential multi-objective programming problem involving these generalized convex functions, respectively.

\section{Preliminaries}

In this section, we first recall some concepts and results related arcwise connected functions. Let $R^{n}$ be the $n$ dimensional Euclidean space and $R^{1}$ be the set of all real numbers. Throughout this paper, the following convention for vectors in $R^{n}$ will be followed:

$$
x<y \text { if and only if } x_{i}<y_{i}, \quad i=1,2, \cdots, n,
$$


$x \leq y$ if and only if $x_{i} \leq y_{i}, \quad i=1,2, \cdots, n$, $x \leq y$ if and only if $x_{i} \leq y_{i}, \quad i=1,2, \cdots, n$, but $x \neq y$, $x \nless y$ is the negation of $x<y$.

Definition 2.1. (See [15]) A subset $X \subset R^{n}$ is said to be an arcwise connected (AC) set, if for every $x \in X$, $u \in X$, there exists a continuous vector-valued functions $H_{x, u}:[0,1] \rightarrow X$, called an arc, such that

$$
H_{x, u}(0)=x, H_{x, u}(1)=u \text {. }
$$

Definition 2.2. (See [15]) Let $f$ be a real-valued function defined on an AC set $X \subset R^{n}$. Then $f$ is said to be an arcwise connected function (CN) if, for every $x \in X, u \in X$, there exists an arc $H_{x, u}$ such that

$$
\left.f\left(H_{x, u}(\theta)\right)\right) \leq(1-\theta) f(x)+\theta f(u) \text {, for } 0 \leq \theta \leq 1
$$

Definition 2.3. (See [13,14]) Let $X \subset R^{n}$ be an AC set, and Let $f$ be a real-valued function defined on $X$. For any $x \in X, u \in X$, the directional derivative of $f$ with respect to $H_{x, u}$ at $\theta=0$ is defined as

$$
\lim _{\theta \rightarrow 0^{+}} \frac{f\left(H_{x, u}(\theta)\right)-f(x)}{\theta},
$$

provided the limit exists and is denoted by $f^{+}\left(H_{x, u}(0)\right)$. If

$$
\lim _{\theta \rightarrow 0^{+}} \frac{H_{x, u}(\theta)-x}{\theta}
$$

exists and it is denoted by $H_{x, u}^{+}(0)$, then vector $H_{x, u}^{+}(0)$ is called directional derivative of $H_{x, u}$ at $\theta=0$.

Consider the following multiobjective programming problem:

$$
\begin{array}{ll}
\text { (MP) } & \min f(x) \\
& \text { s.t. } g(x) \leq 0, x \in X,
\end{array}
$$

where $f: X \rightarrow R^{m}, g: X \rightarrow R^{p}, X$ is a nonempty open AC set of $R^{n}$. Let $\mathrm{F}$ denote the feasible solutions of (MP) assumed to be nonempty, that is

$$
\mathrm{F}=\left\{x \in R^{n}: g(x) \leq 0\right\} .
$$

Definition 2.4. A point $\bar{x} \in X$ is said to be a Pareto efficient solution of problem (MP), if $f(x) \not \leq f(\bar{x})$ for all $x \in X$.

Definition 2.5. A point $\bar{X} \in X$ is said to be a weak Pareto efficient solution of problem (MP), if

$f(x) \not \leq f(\bar{x})$ for all $x \in X$.

Along the lines of $[1,5]$, we now define the following classes of functions.

Definition 2.6. $\left(f_{i}, g_{j}\right), i=1,2, \cdots, m$ and $j=1,2, \cdots, p$, is said to be CN-d-type-I with respect to $H_{x^{*}, x}$, at $x^{*} \in X$ if there exist an arc $H_{x^{*}, x}:[0,1] \rightarrow X$ such that for all $x \in X$,

$$
f_{i}(x)-f_{i}\left(x^{*}\right) \geq f_{i}^{+}\left(H_{x^{*}, x}(0)\right)
$$

and

$$
-g_{j}\left(x^{*}\right) \geq g_{j}^{+}\left(H_{x^{*}, x}(0)\right)
$$

Definition 2.7. $\left(f_{i}, g_{j}\right), i=1,2, \cdots, m$ and $j=1,2, \cdots, p$, is said to be quasi-CN-d-type-I with respect to $H_{x^{*}, x}$ at $x^{*} \in X$ if there exist an arc $H_{x^{*}, x}:[0,1] \rightarrow X$ such that for all $x \in X$,

$$
f_{i}(x) \leq f_{i}\left(x^{*}\right) \Rightarrow f_{i}^{+}\left(H_{x^{*}, x}(0)\right) \leq 0,
$$

and

$$
-g_{j}\left(x^{*}\right) \leq 0 \Rightarrow g_{j}^{+}\left(H_{x^{*}, x}(0)\right) \leq 0 .
$$

Definition 2.8. $\left(f_{i}, g_{j}\right), \quad i=1,2, \cdots, m$ and $j=1,2, \cdots, p$, is said to be pseudo-CN-d-type-I with respect to $H_{x^{*}, x}$ at $x^{*} \in X$ if there exist an arc $H_{x^{*}, x}:[0,1] \rightarrow X$ such that for all $x \in X$,

$$
f_{i}^{+}\left(H_{x^{*}, x}(0)\right) \geq 0 \Rightarrow f_{i}(x) \geq f_{i}\left(x^{*}\right),
$$

and

$$
g_{j}^{+}\left(H_{x^{*}, x}(0)\right) \geq 0 \Rightarrow-g_{j}\left(x^{*}\right) \geq 0 .
$$

Definition 2.9. $\left(f_{i}, g_{j}\right), i=1,2, \cdots, m$ and $j=1,2, \cdots, p$, is said to be quasipseudo-CN-d-type-I with respect to $H_{x^{*}, x}$ at $x^{*} \in X$ if there exist an arc $H_{x^{*}, x}:[0,1] \rightarrow X$ such that for all $x \in X$,

$$
f_{i}(x) \leq f_{i}\left(x^{*}\right) \Rightarrow f_{i}^{+}\left(H_{x^{*}, x}(0)\right) \leq 0,
$$

and

$$
g_{j}^{+}\left(H_{x^{*}, x}(0)\right) \geq 0 \Rightarrow-g_{j}\left(x^{*}\right) \geq 0 .
$$

Definition 2.10. $\left(f_{i}, g_{j}\right), i=1,2, \cdots, m$ and $j=1,2, \cdots, p$, is said to be pseudoquasi-CN-d-type-I with respect to $H_{x^{*}, x}$ at $x^{*} \in X$ if there exist an arc $H_{x^{*}, x}:[0,1] \rightarrow X$ such that for all $x \in X$,

$$
f_{i}^{+}\left(H_{x^{*}, x}(0)\right) \geq 0 \Rightarrow f_{i}(x) \geq f_{i}\left(x^{*}\right),
$$

and

$$
-g_{j}\left(x^{*}\right) \leq 0 \Rightarrow g_{j}^{+}\left(H_{x^{*}, x}(0)\right) \leq 0 .
$$

To show the existence of the CN-d-type-I functions 
we give the following example:

Example 2.1. Define a set $X \subset R^{2}$ as

$$
X=\left\{\left(x_{1}, x_{2}\right): x_{1}^{2}+x_{2}^{2} \geq 1, x_{1}>0, x_{2}>0\right\} .
$$

Then $x$ is an AC set with respect to $H_{x, y}:[0,1] \rightarrow X$ given by

$$
\begin{gathered}
H_{x, y}(\theta)=\left(\left((1-\theta) x_{1}^{2}+\theta y_{1}^{2}\right)^{1 / 2},\left((1-\theta) x_{2}^{2}+\theta y_{2}^{2}\right)^{1 / 2}\right) \\
x=\left(x_{1}, x_{2}\right) \in X, \quad y=\left(y_{1}, y_{2}\right) \in X \forall \theta \in[0,1] .
\end{gathered}
$$

Define $f: X \rightarrow R, g: X \rightarrow R$ as

$$
\begin{gathered}
f(x)= \begin{cases}x_{1}^{2}+x_{2}^{2}, & \text { if } x_{1}>1 \text { and } x_{2}>1 \\
0, & \text { otherwise, }\end{cases} \\
g(x)= \begin{cases}-x_{2}^{2}, & \text { if } x_{1}>1 \text { and } x_{2}>1 \\
0, & \text { otherwise, }\end{cases}
\end{gathered}
$$

$f$ and $g$ are not differentiable at $x^{*}=(1,1) \in X$. However, $f^{+}\left(H_{x^{*}, x}(0)\right)$ and $g^{+}\left(H_{x^{*}, x}(0)\right)$ existed, and they are given by

$$
\begin{gathered}
f^{+}\left(H_{x^{*}, x}(0)\right) \\
= \begin{cases}x_{1}^{2}+x_{2}^{2}, & \text { if both components of } H_{x^{*}, x}>1 \\
0, & \text { otherwise, }\end{cases} \\
g^{+}\left(H_{x^{*}, x}(0)\right)= \begin{cases}-x_{2}^{2}, & \text { if both components of } H_{x^{*}, x}>1 \\
0, & \text { otherwise, }\end{cases}
\end{gathered}
$$

where $x=\left(x_{1}, x_{2}\right)$. It is obviously that $(f, g)$ is CN-dtype-I at $x^{*}=(1,1)$.

\section{Sufficient Optimality Conditions}

In this section, we establish sufficient optimality conditions for a feasible solution $\bar{x}$ to be a weak Pareto efficient solution for (MP) under the CN-d-type-I and pseudoquasi-CN-d-type-I assumptions.

Theorem 3.1. Suppose that there exist a feasible solution $\bar{x} \in \mathrm{F}$ and $\bar{\mu}=\left(\bar{\mu}_{1}, \cdots, \bar{\mu}_{P}\right), \bar{\mu} \geq 0$ such that

$$
\begin{aligned}
& f^{+}\left(H_{\bar{x}, x}(0)\right)+\bar{\mu}^{T} g^{+} \in \geq 0 \quad \forall x \in \mathrm{X} \\
& \bar{\mu}_{j} g_{j}(\bar{x})=0, \quad j=1,2, \cdots, p
\end{aligned}
$$

If $\left(f_{i}, g_{j}\right)$ is CN-d-type-I with respect to $H_{\bar{x}, x}$ at $\bar{x}$, then $\bar{x}$ is a weak Pareto efficient solution for (MP).

Proof Suppose that $\bar{x}$ is not a weak Pareto efficient solution of (MP). Then there is a feasible solution $x$ of (MP) such that

$$
f_{i}(x)<f_{i}(\bar{x}) \text { for any } i=1,2, \cdots, m .
$$

By CN-d-type-I assumption on $f_{i}$, we get $0>f_{i}(x)-f_{i}(\bar{x}) \geq f_{i}^{+}\left(H_{\bar{x}, x}(0)\right)$ for any $i=1,2, \cdots, m$.

Thus,

$$
f_{i}^{+}\left(H_{\bar{x}, x}(0)\right)<0 \text { for any } i=1,2, \cdots, m .
$$

So, we have

$$
\sum_{i=1}^{m} f_{i}^{+}\left(H_{\bar{x}, x}(0)\right)<0 .
$$

It yields from (3.1) that

$$
\sum_{j=1}^{p} \bar{\mu}_{j} g_{j}^{+}\left(H_{\bar{x}, x}(0)\right)>0 .
$$

from CN-d-type-I assumption on $g_{j}$, we get

$$
-g_{j}(\bar{x}) \geq g_{j}^{+}\left(H_{\bar{x}, x}(0)\right) \text {, for any } j=1,2, \cdots, p \text {. }
$$

Since $\bar{\mu} \geq 0$ and Equation (3.2) holds, we can get $0=-\bar{\mu}_{j} g_{j}(\bar{x}) \geq \bar{\mu}_{j} g_{j}^{+}\left(H_{\bar{x}, x}(0)\right)$, for any $j=1,2, \cdots, p$.

Therefore,

$$
\sum_{j=1}^{p} \bar{\mu}_{j} g_{j}^{+}\left(H_{\bar{x}, x}(0)\right) \leq 0,
$$

which contradicts to (3.4).

Theorem 3.2. Suppose that there exist a feasible solution $\bar{x} \in \mathrm{F}$ and $\bar{\mu}=\left(\bar{\mu}_{1}, \cdots, \bar{\mu}_{P}\right), \bar{\mu} \geq 0$ such that (3.1) and (3.2) hold. If $\left(f_{i}, \bar{\mu}_{j} g_{j}\right)$ is pseudoquasi-CN-d-typeI with respect to $H_{\bar{x}, X}$ at $\bar{x}$, then $\bar{x}$ is a weak Pareto efficient solution for (MP).

Proof Since (3.2) holds and $\bar{x} \in \mathrm{F}$, by pseudoquasiCN-d-type-I hypothesis on $\bar{\mu}_{j} g_{j}$ at $\bar{x}$, for all $x \in X$ we have

$$
\bar{\mu}_{j} g_{j}^{+}\left(H_{x^{*}, x}(0)\right) \leq 0
$$

Thus

$$
\sum_{j=1}^{p} \bar{\mu}_{j} g_{j}^{+}\left(H_{x^{*}, x}(0)\right) \leq 0 \text { for all } x \in X .
$$

Let $\bar{x}$ not be a weak Pareto efficient solution for (MP). Then there is a feasible solution $\hat{x}$ for (MP) such that

$$
f_{i}(\hat{x})<f_{i}(\bar{x}) \text { for any } i=1,2, \cdots, m \text {. }
$$

from pseudoquasi-CN-d-type-I hypothesis on $f_{i}$ at $\bar{x}$, it yields

$$
f_{i}^{+}\left(H_{\bar{x}, \hat{x}}(0)\right)<0 \text { for any } i=1,2, \cdots, m .
$$

so,

$$
\sum_{i=1}^{m} f_{i}^{+}\left(H_{\bar{x}, \hat{x}}(0)\right)<0
$$


Combing (3.5) and (3.6), we get

$$
\sum_{i=1}^{m} f_{i}^{+}\left(H_{\bar{x}, \hat{x}}(0)\right)+\sum_{j=1}^{p} \bar{\mu}_{j} g_{j}^{+}\left(H_{\bar{x}, \hat{x}}(0)\right)<0 .
$$

But this is a contradiction to (3.1). The proof is completed.

Remark 3.1. For the functions $f(x)$ and $g(x)$ in Example 2.1, we consider the programming problem (MP). Let $\bar{\mu}=1$, we are easy to get that

$$
f^{+}\left(H_{\bar{x}, \hat{x}}(0)\right)+\bar{\mu}^{T} g^{+}\left(H_{\bar{x}, \hat{x}}(0)\right) \geq 0 \quad \forall x \in X,
$$

which implies that $\bar{x}=(1,1)$ is an optimal solution (is also weak Pareto efficient) of (MP).

\section{Duality Results}

Now, in relation to (MP) we consider the following Mond and Weir type dual under the CN-d-type-I and generalized CN-d-type-I assumptions.

$$
\begin{gathered}
\text { (DMP) } \max f(y)=\left(f_{1}(y), f_{2}(y), \cdots, f_{m}(y)\right) \\
\text { s.t. } \xi^{T} f^{+}\left(H_{y, x}(0)\right)+\mu^{T} g^{+}\left(H_{y, x}(0)\right) \geq 0 \text { for all } y \in \mathrm{F} \\
\sum_{j=1}^{p} \mu_{j} g_{j}(y) \geq 0, \\
\xi>0, \quad \xi=\left(\xi_{1}, \xi_{2}, \cdots, \xi_{m}\right)^{T}, \\
\mu \geq 0, \quad \mu=\left(\mu_{1}, \mu_{2}, \cdots, \mu_{p}\right)^{T} .
\end{gathered}
$$

Theorem 4.1. (Weak Duality) Let $x$ and $(y, \xi, \mu)$ be feasible soltuions for (MP) and (DMP), respectively. Moreover, we assume that any one of the following conditions holds:

a) $\left(f_{i}, g_{j}\right)$ is CN-d-type-I with respect to $H_{y, x}$ at $y$;

b) $\left(f_{i}, \mu_{j} g_{j}\right)$ is pseudoquasi-CN-d-type-I with respect to $H_{y, x}$ at $y$.

Then

$$
f(x) \not \leq f(y) .
$$

Proof Since $(y, \xi, \mu)$ is feasible solution for (DMP), we have

$$
\xi^{T} f^{+}\left(H_{y, x^{\prime}}(0)\right)+\mu^{T} g^{+}\left(H_{y, x^{\prime}}(0)\right) \geq 0 \quad \forall x^{\prime} \in X \geq 0
$$

and (4.2) holds. We proceed by contradiction. Suppose that

$$
f(x) \leq f(y) .
$$

Then, there exists an index $k$ such that

$$
\begin{gathered}
f_{k}(x)<f_{k}(y), \\
f_{i}(x) \leq f_{i}(y) \text { for all } i \neq k .
\end{gathered}
$$

Since $\xi_{i}>0, \quad i=1,2, \cdots, m$, we get

$\xi_{k} f_{k}(x)<\xi_{k} f_{k}(y), \quad \xi_{i} f_{i}(x) \leq \xi_{i} f_{i}(y)$ for all $i \neq k$.

Thus,

$$
\sum_{i=1}^{m} \xi_{i} f_{i}(x)<\sum_{i=1}^{m} \xi_{i} f_{i}(y) .
$$

by condition (a), we get

$$
f_{i}(y)-f_{i}(x) \geq f_{i}^{+}\left(H_{y, x}(0)\right)
$$

and

$$
-g_{j}(y) \geq g_{j}^{+}\left(H_{y, x}(0)\right) .
$$

Therefore, we can get

$$
\sum_{i=1}^{m} \xi_{i} f_{i}(x)-\sum_{i=1}^{m} \xi_{i} f_{i}(y) \geq \sum_{i=1}^{m} \xi_{i} f_{i}^{+}\left(H_{y, x}(0)\right)
$$

and

$$
-\sum_{j=1}^{p} \mu_{j} g_{j}(y) \geq \sum_{j=1}^{p} \mu_{j} g_{j}^{+}\left(H_{y, x}(0)\right) .
$$

Combing (4.2), (4.7), (4.8) and (4.9), we get

$$
\sum_{i=1}^{m} \xi_{i} f_{i}^{+}\left(H_{y, x}(0)\right)<0,
$$

and

$$
\sum_{j=1}^{p} \mu_{j} g_{j}^{+}\left(H_{y, x}(0)\right) \leq 0
$$

so

$$
\sum_{i=1}^{m} \xi_{i} f_{i}^{+}\left(H_{y, x}(0)\right)+\sum_{j=1}^{p} \mu_{i} g_{j}^{+}\left(H_{y, x}(0)\right)<0
$$

which contradicts (4.6).

By condition (b), noticing that (4.2) holds, with the similar argument as that of Theorem 3.2, we can get

$$
\sum_{i=1}^{m} \xi_{i} f_{i}^{+}\left(H_{y, x}(0)\right)<0,
$$

and

$$
\sum_{j=1}^{p} \mu_{j} g_{j}^{+}\left(H_{y, x}(0)\right) \leq 0 .
$$

The above two inequalities imply (4.10), again a contradiction to (4.6). This completes the proof.

Theorem 4.2. Suppose that there exist feasible solutions $\bar{x}$ and $(\bar{y}, \bar{\xi}, \bar{\mu})$ for (MP) and (DMP), respectively, such that 


$$
f_{i}(\bar{x})=f_{i}(\bar{y}), \quad i=1,2, \cdots, m .
$$

Moreover, we assume that the hypotheses of Theorem 4.1 hold at $\bar{y}$, then $\bar{x}$ is a Pareto efficient solution for (MP).

Proof For any feasible solution $x$ for (MP), we get from Theorem 4.1 that

$$
f(x) \not \leq f(y) .
$$

Suppose that $\bar{X}$ is not a Pareto efficient solution for (MP). Then, there exist a feasible solution $\hat{x}$ for (MP) and an index $k$ such that

$$
f_{k}(\hat{x})<f_{k}(\bar{x}), \quad f_{k}(\hat{x}) \leq f_{k}(\bar{x}) \text { for all } i \neq k .
$$

Using condition (4.11), we get

$$
f_{k}(\hat{x})<f_{k}(\bar{y}), \quad f_{k}(\hat{x}) \leq f_{k}(\bar{y}) \text { for all } i \neq k \text {. }
$$

This contradicts to Theorem 4.1.

Theorem 4.3. (Converse Duality) Let $(y, \xi, \mu)$ be a Pareto efficient solution for (DMP). Moreover, we assume that the hypotheses of Theorem 4.1 hold at $y$, then $y$ is a Pareto efficient solution for (MP).

Proof We proceed by contradiction. Suppose that $y$ is not a Pareto efficient solution for (MP), that is, there exist $x \in \mathrm{F}$ and an index $k$ such that

$$
f_{k}(x)<f_{k}(y), \quad f_{i}(x) \leq f_{i}(y) \text { for all } i \neq k \text {. }
$$

If any one of the hypotheses of Theorem 4.1 holds, it yields in light of Theorem 4.1 that (4.5) is satisfied. This leads to the similar contradiction as in the proof of Theorem 4.1.

\section{References}

[1] M. A. Hanson and B. Mond, "Necessary and Sufficient Conditions in Constraint Optimization," Mathematical Programming, Vol. 37, No. 1, 1987, pp. 51-58. doi:10.1007/BF02591683

[2] B. Aghezzaf and M. Hachimi, "Generalized Invexity and Duality in Multiobjective Programming Problems," Journal of Global Optimization, Vol. 18, No. 1, 2000, pp. 91-101. doi:10.1023/A:1008321026317

[3] M. Hachimi and B. Aghezzaf, "Sufficiency and Duality in Differentiable Multiobjective Programming Involving Generalized Type I Functions," Journal of Mathematical Analysis and Applications, Vol. 296, No. 2, 2004, pp. 382-392. doi:10.1016/j.jmaa.2003.12.042

[4] M. Hachimi and B. Aghezzaf, "Sufficiency and Duality in Nondifferentiable Multiobjective Programming Involving Generalized Type I Functions," Journal of Mathematical Analysis and Applications, Vol. 319, No. 1, 2006, pp. 110-123. doi:10.1016/j.jmaa.2005.02.064

[5] R. N. Kaul, S. K. Suneja and M. K. Srivastava, "Optimality Criteria and Duality in Multiple Objective Optimization Involving Generalized Invexity," Journal of Optimi- zation Theory and Applications, Vol. 80, No. 3, 1994, pp. 465-482. doi:10.1007/BF02207775

[6] S. K. Mishra, G. Giorgi and S. Y. Wang, "Duality in Vector Optimization in Banach Spaces with Generalized Convexity,” Journal of Global Optimization, Vol. 29, No. 4, 2004, pp. 415-424. doi:10.1023/B:JOGO.0000047911.03061.88

[7] S. K. Mishra and M. A. Noor, "Some Nondifferentiable Multi-Objective Programming Problems," Journal of Mathematic Analysis and Applications, Vol. 316, No. 2, 2006, pp. 472-482. doi:10.1016/j.jmaa.2005.04.067

[8] S. K. Mishra, S. Y. Wang and K. K. Lai, "Multiple Objective Fractional Programming Involving Semilocally Type I-Preinvex and Related Functions," Journal of Mathematic Analysis and Applications, Vol. 310, No. 2, 2005, pp. 626-640.

[9] A. Mehra and D. Bhatia, "Optimality and Duality for Minmax Problems Involving Arcwise Connected and Generalized Arcwise Connected Functions,” Journal of Mathematical Analysis and Applications, Vol. 231, No. 2, 1999, pp. 425-445. doi:10.1006/jmaa.1998.6231

[10] N. G. Rueda, M. A. Hanson and C. Singh, "Optimality and Duality with Generalized Convexity,” Journal of Optimization Theory and Applications, Vol. 86, No. 2, 1995, pp. 491-500. doi:10.1007/BF02192091

[11] G. L. Yu and S. Y. Liu, "Some Vector Optimization Problems in Banach Spaces with Generalized Convexity,” Computers and Mathematics with Applications, Vol. 54, No. 11-12, 2007, pp. 1403-1410. doi:10.1016/j.camwa.2007.05.002

[12] G. L. Yu and S. Y. Liu, "Optimality for $(h, \phi)$-Multiobjective Programming Involving Generalized Type-I Functions,” Journal of Global Optimization, Vol. 41, No. 1, 2008, pp. 147-161. doi:10.1007/s10898-007-9196-3

[13] M. Avriel and I. Zang, "Generalized Arcwise-Connected Functions and Characterizations of Local-Global Minimum Properties," Journal of Optimization Theory and Applications, Vol. 32, No. 4, 1980, pp. 407-425. doi:10.1007/BF00934030

[14] C. Singh, "Elementary Properties of Arcwise Connected Set and Functions," Journal of Optimization Theory and Applications, Vol. 41, 1990, pp. 85-103.

[15] R. N. Mukherjee and S. R. Yadav, "A Note on Arcwise Connected Sets and Functions," Bulletin of the Australian Mathematical Society, Vol. 31, No. 3, 1985, pp. 369-375. doi:10.1017/S0004972700009333

[16] D. Bhatia and A. Mehra, "Optimality and Duality Involving Arcwise Connected Generalized Connected Functions," Journal of Optimization Theory and Applications, Vol. 100, No. 1, 1999, pp. 181-194. doi:10.1023/A:1021725200423

[17] N. G. Rueda and M. A. Hanson, "Optimality Criteria in Mathematical Programming Involving Generalized Invexity," Journal of Mathematical Analysis and Applications, Vol. 130, No. 2, 1998, pp. 375-385. doi:10.1016/0022-247X(88)90313-7 
[18] S. Davar and A. Mehra, "Optimality and Duality for Fractional Programming Problems Involving Arcwise Connected Functions and Their Applications,” Journal of
Mathematical Analysis and Applications, Vol. 263, No. 2, 2001, pp. 666-682. doi:10.1006/jmaa.2001.7651 